\title{
Modified signals for supersymmetry in the NMSSM with a singlino-like LSP
}

\author{
Debottam Das, ${ }^{a}$ Ulrich Ellwanger $^{a}$ and Ana M. Teixeira ${ }^{b}$ \\ ${ }^{a}$ Laboratoire de Physique Théorique, UMR 8627, CNRS and Université de Paris-Sud, \\ Bât. 210, 91405 Orsay, France \\ ${ }^{b}$ Laboratoire de Physique Corpusculaire, CNRS/IN2P3 - UMR 6533, \\ Campus des Cézeaux, 24 Av. des Landais, F-63171 Aubière Cedex, France \\ E-mail: debottam.das@th.u-psud.fr, ulrich.ellwanger@th.u-psud.fr, \\ ana.teixeira@clermont.in2p3.fr
}

ABSTRACT: In the framework of the NMSSM with a singlino-like LSP, we study quantitatively the impact of the additional bino $\rightarrow$ singlino cascade on the efficiencies in several search channels for supersymmetry of the ATLAS and CMS collaborations. Compared to the MSSM, the additional cascade reduces the missing transverse energy, but leads to additional jets or leptons. For the NMSSM benchmark lines which generalize cMSSM benchmark points, the efficiencies in the most relevant $2 / 3$ jet + missing energy search channels can drop by factors $\sim 1 / 3$ to $\sim 1 / 7$, and can reduce the present lower bounds on $M_{1 / 2}$ by as much as $\sim 0.9-0.75$ in the NMSSM for large bino-singlino mass differences. The larger efficiencies in multijet or multilepton search channels are not strong enough to affect this conclusion. In the fully constrained cNMSSM, sparticle decay cascades via the lightest stau can lead to signal cross sections in multilepton and $2 \tau$ search channels which are potentially visible at the LHC with $7 \mathrm{TeV}$ center of mass energy.

KEYWORDS: Supersymmetry Phenomenology

ARXIV EPRINT: 1202.5244 


\section{Contents}

1 Introduction 1

2 The NMSSM with a singlino-like LSP 4

3 Monte Carlo simulations and search channels $\quad 8$

4 Results $\quad 10$

$\begin{array}{lll}4.1 & \text { Benchmark line 10.1.1 } & 10\end{array}$

4.2 Benchmark line 10.4.1 14

$\begin{array}{lll}4.3 & \text { Benchmark line 40.2.1 } & 16\end{array}$

$\begin{array}{lll}4.4 & \text { The cNMSSM } & 18\end{array}$

5 Conclusions and outlook $\quad 20$

\section{Introduction}

The Next-to-Minimal Supersymmetric Standard Model (NMSSM) [1] is the simplest supersymmetric (SUSY) extension of the Standard Model with a scale invariant superpotential, i.e. where the soft SUSY breaking terms are the only dimensionful parameters. A supersymmetric Higgs mass term $\mu$, as required in the MSSM, is generated dynamically by a vacuum expectation value (vev) of a gauge singlet (super-)field $S$, and is automatically of the order of the supersymmetry breaking scale. The attractive features of the MSSM are preserved, like a solution of the hierarchy problem, the unification of the running gauge coupling constants at a Grand Unification (GUT) scale, and a dark matter candidate in the form of a stable lightest SUSY particle (LSP).

Using data from $1-2 \mathrm{fb}^{-1}$ of integrated luminosity at the LHC at $7 \mathrm{TeV}$ center of mass (c.m.) energy, searches for supersymmetry by the ATLAS [2-7] and CMS [8-18] collaborations have not led to the discovery of signals of supersymmetric particles.

However, already within the MSSM, the interpretation of the absence (or presence) of signals in the many possible channels depends on the soft SUSY breaking terms: Assuming R-parity conservation and a neutralino-like LSP, missing transverse energy $E_{T}^{\text {miss }}$ together with high $p_{T}$ jets and/or leptons are used as search criteria. These signatures depend on the decay cascades of the initially produced squarks and gluinos (dominant at a hadron collider), which depend on the spectrum and the couplings of sparticles (squarks, gluino, sleptons, charginos and neutralinos), and hence on the soft SUSY breaking terms.

A popular choice for the soft SUSY breaking terms is the constrained MSSM (cMSSM) where scalar masses $m_{0}$, gaugino masses $M_{1 / 2}$ and trilinear couplings $A_{0}$ are assumed to be universal at the GUT scale. In the cMSSM, the lightest neutralino $\chi_{1}^{0}$ is typically the 
LSP, and bino-like in most of the parameter space (apart from very small values of $m_{0}$, or very large values of both $m_{0}$ and $\left.M_{1 / 2}\right) \cdot \chi_{1}^{0}$ appears as one of the final states in every sparticle decay cascade, and is responsible for the missing transverse energy.

Assuming fixed values for $A_{0}$ and $\tan \beta$ (the ratio of the two Higgs vevs $\left\langle H_{u}\right\rangle /\left\langle H_{d}\right\rangle$ ), the ATLAS and CMS collaborations have deduced lower bounds on $m_{0}$ and $M_{1 / 2}$ in the $m_{0}-M_{1 / 2}$ plane in the cMSSM. Recently, the absence of signals has been interpreted within more general scenarios of soft SUSY breaking terms as the phenomenological MSSM [19-21] and anomaly mediation [22]. In the present paper, we perform a first study of possible modifications of signals for supersymmetric particles in the framework of a generalization of the cMSSM towards the NMSSM.

The additional gauge singlet superfield $\hat{S}$ in the NMSSM leads to additional physical states in the CP-even and CP-odd Higgs sectors, as well as an additional singlino-like neutralino. The impact of the additional Higgs states can be very important for Higgs searches (see [23] for a review), but in the present paper we concentrate on the possible impact of the additional singlino-like neutralino on searches for supersymmetry. This becomes particularly relevant in regions of the NMSSM parameter space where the singlinolike neutralino is the LSP $\chi_{1}^{0}$ : Then the singlino-like neutralino appears as one of the final states in every sparticle decay cascade.

However, the couplings of the singlino-like neutralino to all other sparticles are typically very small. Then the branching ratios for decays of all sparticles into the singlino-like LSP $\chi_{1}^{0}$ are small, with the exception of the NLSP, which can decay only into $\chi_{1}^{0}$ if R-parity is conserved. Hence sparticle decay cascades will evolve as in the MSSM, with an additional final decay

$$
\chi_{2}^{0} \rightarrow \chi_{1}^{0}+X
$$

(if the next-to-lightest neutralino $\chi_{2}^{0}$ is the next-to-LSP (NLSP) which is, however, not necessarily the case in the NMSSM). Depending on the mass difference

$$
\Delta_{M}=M_{\chi_{2}^{0}}-M_{\chi_{1}^{0}}
$$

and on the nature of the decay products $X$ in (1.1), this additional decay of the NLSP will generally reduce $E_{T}^{\text {miss }}$, but will lead to more jets and/or leptons. Hence it affects all signatures used in searches for supersymmetry. A priori, it is not clear how the reduction of $E_{T}^{\text {miss }}$ and the additional jets and/or leptons affect the efficiencies in the various SUSY search channels. A quantitative analysis of the modifications of the efficiencies in the NMSSM, for some relevant search channels for supersymmetry (without and with leptons), is the purpose of the present paper.

Like in the general MSSM, many different scenarios for the soft SUSY breaking terms are possible in the NMSSM. We find it useful to compare NMSSM and MSSM scenarios which are as close as possible: Similar squark, slepton and gaugino spectra, but with an additional singlino-like LSP in the NMSSM. For convenience, we compare a semiconstrained version of the NMSSM (sNMSSM) to the cMSSM: We choose the same values for $m_{0}, M_{1 / 2}, A_{0}$ and $\tan \beta$ as for some benchmark points of the cMSSM, but allow for nonuniversal singlet-specific soft SUSY breaking terms $m_{S}^{2}, A_{\lambda}$ and $A_{\kappa}$ (see the next section) such that the singlino-like neutralino is the LSP $\chi_{1}^{0}$, and the extended Higgs sector is in agreement with constraints from LEP. 
The advantage of this approach is that, for each choice of $m_{0}, M_{1 / 2}, A_{0}$ and $\tan \beta$, we can compare directly the efficiencies in various channels (after appropriate cuts) between the cMSSM and the sNMSSM. This allows to estimate to which extent the additional singlino-like neutralino affects present or future limits or excesses in the $m_{0}-M_{1 / 2}$ plane in comparison to the cMSSM as a function of $\Delta_{M}$, without the need for a novel analysis of backgrounds and systematic errors.

Since we cannot study the complete $m_{0}-M_{1 / 2}$ plane, we consider three benchmark points of the cMSSM as defined in [24], with not too large values of $m_{0}$ and $M_{1 / 2}$ (presently not excluded, but of possible future relevance for the LHC at $7 \mathrm{TeV}$ c.m. energy). For each point we vary the NMSSM-specific parameters in a domain where the singlino-like neutralino is the $\operatorname{LSP}\left(\chi_{1}^{0}\right)$, allowing $\Delta_{M}$ to vary within the largest possible range complying with constraints from LEP2 on the Higgs sector. Thus each benchmark point of the cMSSM is promoted to a benchmark line in the sNMSSM. We then study the ratios $R$ of efficiencies sNMSSM/cMSSM for 5 search channels of the ATLAS jets + missing transverse momentum (0 leptons) analysis in [2], and 4 search channels of the ATLAS multijet + missing transverse momentum (0 leptons) analysis in [4], always as function of $\Delta_{M}$. (For our purpose it is useful that the lower bounds in the $m_{0}-M_{1 / 2}$ plane are given for each search channel separately on the web page [25].)

Actually these ratios $R$ always tend towards 1 in the limit $\Delta_{M} \rightarrow 0$ : Then the complete (missing) energy is transferred from $\chi_{2}^{0}$ to $\chi_{1}^{0}$ in the decay (1.1), and no energy remains to generate high $p_{T}$ jets or leptons from the decay products $X$. However, for larger $\Delta_{M}$ we find that, after cuts corresponding to the most sensitive 2/3-jet, 0 lepton search channels, the efficiencies in the sNMSSM can be smaller by a factor $\sim 1 / 7$ as compared to the cMSSM! Simultaneously, in the less sensitive multijet search channels the efficiencies in the sNMSSM would be larger than in the cMSSM.

Since the additional decay products $X$ in (1.1) can consist of leptons, it is also interesting to study search channels of the CMS multilepton analysis in [17] for the sNMSSM. Here, however, the corresponding cMSSM efficiencies are so small that it is not meaningful to give ratios of efficiencies sNMSSM/cMSSM; hence we give estimates for absolute signal cross sections (production cross sections $\times$ efficiencies) for those values of $\Delta_{M}$ which correspond to the largest efficiencies. The absolute signal cross sections allow to estimate the future discovery potential in these channels.

The phenomenologically allowed region in the $m_{0}-M_{1 / 2}$ plane in the sNMSSM is actually somewhat larger than in the cMSSM: Small values for $m_{0}$ would lead to a stau $\left(\tilde{\tau}_{1}\right)$ LSP in the cMSSM, while in the sNMSSM the singlino-like neutralino can be lighter than the $\tilde{\tau}_{1}$ even for $m_{0} \rightarrow 0$. In particular, this scenario is always realised in the fully constrained cNMSSM [26, 27], where the soft singlet mass term $m_{S}$ satisfies $m_{S}=m_{0}$ at the GUT scale, and $m_{S}$ must be small in order to allow for a non-vanishing singlet vev. The sparticle decay cascades in the cNMSSM are quite peculiar: Since the singlino-like LSP couples only very weakly to all MSSM-like sparticles, the latter decay first into the $\tilde{\tau}_{1}$ NLSP. Only subsequently does the $\tilde{\tau}_{1}$ NLSP decay into the singlino-like LSP $\chi_{1}^{0}$, leading finally to $2 \tau$ 's in the final state of each sparticle decay chain. However, the $\tau$ 's from the final $\tilde{\tau}_{1} \rightarrow \chi_{1}^{0}$ decay are always quite soft due to the small $\tilde{\tau}_{1}-\chi_{1}^{0}$ mass difference. Hence, 
only the more energetic $\tau$ 's from the sparticle $\rightarrow \tilde{\tau}_{1}+\tau$ decay constitute a visible particular feature of the cNMSSM. (In the cMSSM in the stau-coannihilation region, $\tau$ production from decays into and of the $\tilde{\tau}_{1}$ NLSP can also be expected, although not as frequently as in the cNMSSM.)

For the LHC at $14 \mathrm{TeV}$ c.m. energy, appropriate cuts for searches for the cNMSSM (and ways to distinguish it from the cMSSM) have been proposed and studied in [28]. Using data from $1 \mathrm{fb}^{-1}$ of integrated luminosity at the LHC at $7 \mathrm{TeV}$ c.m. energy, $2 \tau$ channels have been analysed by CMS in [12].

In what follows, we also perform some analyses of the two cNMSSM benchmark points with the lowest values of $M_{1 / 2}$, defined in [24]. (LEP bounds on the Higgs sector imply $M_{1 / 2} \gtrsim 520 \mathrm{GeV}$ in the cNMSSM $[26,27]$.) As done for the comparison of the sNMSSM with the cMSSM, we study the ratios of efficiencies for 5 search channels of the ATLAS jets + missing transverse momentum (0 leptons) analysis in [2] (using similar realistic cMSSM points with small, but non-vanishing values of $m_{0}$, which are not excluded by present searches). In these search channels, the efficiencies in the cNMSSM and the cMSSM turn out to be quite similar. In addition we give estimates for absolute signal cross sections for 4 search channels of the CMS multilepton analysis in [17] and the CMS $2 \tau$ channels [12]. The corresponding efficiencies in the cMSSM would be very small, which could allow to distinguish these models in the future.

The remainder of the paper is organised as follows: In the next section we discuss the NMSSM with a singlino-like neutralino, define 3 different benchmark lines of the sNMSSM, and 2 benchmark points of the cNMSSM. In section 3 we describe the tools used for our Monte Carlo study, and the cuts used for the ATLAS/CMS search channels. Section 4 contains our main results. For each of the 3 benchmark lines of the sNMSSM, we present first the branching ratios for the decay (1.1) into the additional final states $X$ as function of $\Delta_{M}$. We then give the ratios of efficiencies sNMSSM/cMSSM for different supersymmetry search channels used by ATLAS, and estimates for the signal cross sections for 4 search channels of the CMS multilepton analysis for values of $\Delta_{M}$ corresponding to the largest efficiencies. For the cNMSSM points we provide, in addition, estimates for absolute signal cross sections for the CMS $2 \tau$ channels. Conclusions and an outlook are presented in section 5 .

\section{The NMSSM with a singlino-like LSP}

The NMSSM differs from the MSSM due to the presence of the gauge singlet superfield $\hat{S}$. In the simplest realisation of the NMSSM, the Higgs mass term $\mu \hat{H}_{u} \hat{H}_{d}$ in the MSSM superpotential $W_{M S S M}$ is replaced by the coupling $\lambda$ of $\hat{S}$ to $\hat{H}_{u}$ and $\hat{H}_{d}$, and a self-coupling $\kappa \hat{S}^{3}$. Hence, in this version the superpotential $W_{\text {NMSSM }}$ is scale invariant, and given by:

$$
W_{\mathrm{NMSSM}}=\lambda \hat{S} \hat{H}_{u} \cdot \hat{H}_{d}+\frac{\kappa}{3} \hat{S}^{3}+\ldots,
$$

where the dots denote the Yukawa couplings of $\hat{H}_{u}$ and $\hat{H}_{d}$ to the quarks and leptons as in the MSSM. Once the scalar component of $\hat{S}$ develops a vev $s$, the first term in $W_{\text {NMSSM }}$ generates an effective $\mu$-term with

$$
\mu_{\mathrm{eff}}=\lambda s .
$$


The NMSSM-specific soft SUSY breaking terms consist of a mass term for the scalar components of $\hat{S}$, and trilinear interactions associated to the terms in $W_{\mathrm{NMSSM}}$ :

$$
-\mathcal{L}_{\mathrm{NMSSM}}^{\mathrm{Soft}}=m_{S}^{2}|S|^{2}+\left(\lambda A_{\lambda} H_{u} \cdot H_{d} S+\frac{1}{3} \kappa A_{\kappa} S^{3}\right)+\text { h.c. } .
$$

The neutral CP-even Higgs sector contains 3 states $H_{i}$, which are mixtures of the CPeven components of the superfields $\hat{H}_{u}, \hat{H}_{d}$ and $\hat{S}$. Their masses are described by a $3 \times 3$ mass matrix $\mathcal{M}_{H i j}^{2}$. The neutral CP-odd Higgs sector contains 2 physical states $A_{i}$, whose masses are described by a $2 \times 2$ mass matrix $\mathcal{M}_{A i j}^{2}$. In the neutralino sector we have 5 states $\chi_{i}^{0}$, which are mixtures of the bino $\tilde{B}$, the neutral wino $\tilde{W}^{3}$, the neutral higgsinos from the superfields $\hat{H}_{u}$ and $\hat{H}_{d}$, and the singlino from the superfield $\hat{S}$. Their masses are described by a $5 \times 5$ mass matrix $\mathcal{M}_{\chi^{0} i j}$.

Subsequently, it is of interest to consider the singlet-like components of these mass matrices (given in [1]), for simplicity in the typical range $s \gg v_{u}, v_{d}$, where $v_{u}, v_{d}$ are the vevs of $H_{u}, H_{d}$ :

$$
\begin{aligned}
\mathcal{M}_{H 33}^{2} & \sim \kappa s\left(A_{\kappa}+4 \kappa s\right) \\
\mathcal{M}_{A 22}^{2} & \sim-3 \kappa s A_{\kappa} \\
\mathcal{M}_{\chi^{0} 55} & =2 \kappa s
\end{aligned}
$$

From the above one easily derives

$$
\mathcal{M}_{\chi^{0} 55}^{2} \sim \mathcal{M}_{H 33}^{2}+\frac{1}{3} \mathcal{M}_{A 22}^{2} .
$$

Since both matrix elements $\mathcal{M}_{H 33}^{2}$ and $\mathcal{M}_{A 22}^{2}$ must be positive, one can conclude that none of them can be large if $\mathcal{M}_{\chi^{0} 55}$ is small, and notably that $\mathcal{M}_{H 33}^{2}<\mathcal{M}_{\chi^{0} 55}^{2}$.

In general, these matrix elements differ from the physical masses due to mixing effects. However, the mixing angles are small for small off-diagonal singlet-doublet matrix elements (which are proportional to $\lambda$ ) or large mass differences, and for not too large $\lambda$ the physical masses in the singlet sector are quite close to the above expressions. Hence, a light singletlike neutralino is always accompanied by a lighter singlet-like CP-even Higgs boson, and the singlet-like CP-odd Higgs boson is maximally $\sim \sqrt{3}$ times as heavy as the singlet-like neutralino, but typically lighter. This has important consequences for the possible final states $X$ in the decay (1.1), where these Higgs states are often kinematically allowed and constitute possible 2-body decay channels $\left(X \equiv H_{S}\right.$ or $X \equiv A_{S}$ where the index $S$ denotes a mostly singlet-like state) of the bino-like neutralino $\chi_{2}^{0}$.

These considerations are valid for the general NMSSM with a scale invariant superpotential. As stated in the Introduction, we consider subsequently the semi-constrained sNMSSM where the non-singlet scalar masses, non-singlet trilinear couplings and gaugino masses are universal at the GUT scale with values denoted by $m_{0}, A_{0}$ and $M_{1 / 2}$, respectively. The remaining parameters $\lambda, \kappa, m_{S}^{2}, A_{\lambda}$ and $A_{\kappa}$ of the SNMSSM are chosen as follows: First, we choose a small value for $\lambda$, implying that the singlet-like Higgs bosons and the singlino-like neutralino couple only weakly to all other particles and sparticles. Then the light singlet-like CP-even Higgs boson is compatible with LEP constraints (due 
to its small coupling to the $Z$ boson), and the choice of the sNMSSM specific parameters has little impact on the MSSM-like Higgs and sparticle spectrum. This ensures that the differences of the efficiencies with respect to the cMSSM are only due to the presence of the singlino-like neutralino, and not due to modifications of e.g. the higgsino and/or Higgs spectra: The MSSM-like parameters $\mu$ and $M_{A}$ are kept fixed, which determines implicitely the values of $m_{S}^{2}$ and $A_{\lambda}$ in the sNMSSM. (Fixing $\mu_{\mathrm{eff}}$ for fixed $\lambda$ implies from (2.2) that the vev $s$ is fixed as well.) Then we vary $\kappa$ and $A_{\kappa}$ such that the singlino-like neutralino mass (2.6) is below the bino-like neutralino mass, and the matrix elements (2.4) and (2.5) remain positive. Positive singlet-like Higgs masses compatible with LEP constraints imply actually lower bounds on the singlino-like neutralino mass, but these lower bounds still allow for $\Delta_{M}$ to vary over a wide range.

As in the cMSSM, the dark matter relic density does not generally comply with the WMAP bounds for generic points in the sNMSSM $m_{0}-M_{1 / 2}$ plane, being too large for a singlino-like LSP. As in [24], one could assume a deviation from standard Big-Bang cosmology to reduce the relic density, or a small R-parity violation that renders the LSP unstable. Alternatively, one could modify the parameters in the Higgs sector (and choose a larger value of $\lambda$ ), such that a CP-even or CP-odd s-channel resonance is available for LSP pair annihilation. Such modifications would have little impact on our subsequent results.

As stated in the Introduction, we define three benchmark lines in the sNMSSM for which the values for $m_{0}, M_{1 / 2}, A_{0}$ and $\tan \beta$ correspond to three benchmark points in the cMSSM defined in [24]: 10.1.1, 10.4.1 and 40.2.1. These correspond to different (but not too large) values of $m_{0}$ and $M_{1 / 2}$, with the first number (10 or 40) denoting the value of $\tan \beta$. In each case we fix a small value of $\lambda$, use the cMSSM values for $\mu_{\text {eff }}$ and $M_{A}$, and vary $\kappa$ and $A_{\kappa}$ such that $\Delta_{M}=M_{\chi_{2}^{0}}-M_{\chi_{1}^{0}}$ varies from 0 to a maximal value determined by LEP constraints on the Higgs sector. The singlet-like CP-even and CP-odd Higgs masses vary somewhat with varying $\kappa$ and $A_{\kappa}$, but are always small as shown above. All MSSMlike sparticle properties (notably the squark and gluino masses) are practically constant along the sNMSSM benchmark lines. Hence the sparticle production cross sections in the sNMSSM remain the same as in the cMSSM by construction; these are not affected by the additional singlino-like neutralino (or Higgs) states.

In table 1 we indicate the most relevant properties of the three benchmark lines: First the cMSSM-like parameters $m_{0}, M_{1 / 2}, A_{0}, \tan \beta, \mu_{\text {eff }}$ and $M_{A}$, and the gluino mass $m_{\tilde{g}}$, the average squark masses $\left\langle m_{s q}\right\rangle$ of the first families, and the bino-like neutralino mass $M_{\chi_{2}^{0}}$ (note that $\chi_{2}^{0}$ is the NLSP in the sNMSSM). Subsequently we give the ranges of the NMSSM-specific parameters, the singlino-like neutralino mass $M_{\chi_{1}^{0}}$ and the SM-like and singlet-like Higgs masses $M_{H_{\mathrm{SM}}}, M_{H_{S}}$ and $M_{A_{S}}$, respectively. $\left(M_{H_{\mathrm{SM}}}\right.$ can be larger or smaller than $M_{H_{S}}$. The sparticle and Higgs spectrum is obtained with the help of the code NMSPEC [29] within the version 3.0.2 of NMSSMTools [30, 31].) Finally we provide the total sparticle production cross section $\sigma_{\text {Tot }}$ at the $7 \mathrm{TeV}$ LHC as obtained by Prospino (at next-to-leading order) [32-34].

In addition, we study two points of the fully constrained cNMSSM [26, 27] where the singlet-specific soft SUSY breaking terms $m_{S}, A_{\lambda}$ and $A_{\kappa}$ are also respectively given by $m_{0}$, $A_{0}$ at the GUT scale, and the $\tilde{\tau}_{1}$ is the NLSP. To comply with a dark matter relic density 


\begin{tabular}{|c|c|c|c|}
\hline Point: & 10.1 .1 & 10.4 .1 & 40.2 .1 \\
\hline$M_{1 / 2}$ & 500 & 350 & 450 \\
\hline$m_{0}$ & 125 & 750 & 550 \\
\hline$A_{0}$ & 0 & 0 & -500 \\
\hline $\tan \beta$ & 10 & 10 & 40 \\
\hline$\mu_{\text {eff }}$ & 635 & 465 & 645 \\
\hline$M_{A}$ & 720 & 895 & 710 \\
\hline$m_{\tilde{g}}$ & 1145 & 870 & 1065 \\
\hline$\left\langle m_{s q}\right\rangle$ & 1030 & 1040 & 1080 \\
\hline$M_{\chi_{2}^{0}}$ & 205 & 143 & 187 \\
\hline \hline$\lambda$ & $10^{-3}$ & 0.013 & $10^{-3}$ \\
\hline$\kappa$ & $-1.6 \cdot 10^{-4} \ldots-2 \cdot 10^{-5}$ & $-2 \cdot 10^{-3} \ldots-8.7 \cdot 10^{-4}$ & $1.8 \cdot 10^{-5} \ldots 1.4 \cdot 10^{-4}$ \\
\hline$A_{\kappa}$ & $0.7 \ldots 1.6$ & $0 \ldots 150$ & $-7 \ldots-4.2$ \\
\hline$M_{\chi_{1}^{0}}$ & $25 \ldots 205$ & $50 \ldots 143$ & $23 \ldots 187$ \\
\hline$M_{H_{\mathrm{SM}}}$ & $\sim 115$ & $115 \ldots 117$ & $\sim 117$ \\
\hline$M_{H_{S}}$ & $25 \ldots 205$ & $55 \ldots 89$ & $21 \ldots 186$ \\
\hline$M_{A_{S}}$ & $8 \ldots 20$ & $5 \ldots 160$ & $87 \mathrm{fb}$ \\
\hline$\sigma_{\text {Tot }}$ & $82 \mathrm{fb}$ & $300 \mathrm{fb}$ & \\
\hline
\end{tabular}

Table 1. Parameters, some sparticle and Higgs masses, and the total sparticle production cross section for three sNMSSM benchmark lines corresponding to the cMSSM benchmark points 10.1.1, 10.4.1 and 40.2.1 from [24] (masses in GeV, rounded to $5 \mathrm{GeV}$ accuracy except for $M_{\chi_{2}^{0}}$ and the Higgs masses).

compatible with WMAP constraints, the singlino-like $\chi_{1}^{0}$ must be a few GeV lighter than the $\tilde{\tau}_{1}$ which defines a nearly unique line in the $M_{1 / 2}, A_{0}, \tan \beta$ parameter space $[26,27]$ (taking $m_{0}=0, M_{1 / 2}>520 \mathrm{GeV}$ and $\lambda=10^{-3}$, such that the CP-even Higgs sector complies with LEP constraints).

In [24], benchmark points cNMSSM.1 and cNMSSM.2 (amongst others) have been defined. In the cMSSM parameter space, similar points can be found in the so-called staucoannihilation region: For identical values of $M_{1 / 2}, A_{0}$ and $\tan \beta$, it suffices to choose small non-vanishing values for $m_{0}$ such that the $\tilde{\tau}_{1}$ mass is just above the bino mass, which leads again to a good relic density and a sparticle spectrum which is otherwise very close to the cNMSSM. We find it appropriate to compare efficiencies in the mostly used jets + missing energy channels for the points cNMSSM.1 and cNMSSM.2 to efficiencies for similar points in the cMSSM, denoted here by cMSSM.1 and cMSSM.2. In table 2 we give the most relevant properties of these benchmark points cNMSSM.1 and cNMSSM.2, together with the points cMSSM.1 and cMSSM.2 for which $m_{0}$ and hence the squark/slepton masses are slightly larger. (For the cNMSSM points $\chi_{1}^{0}$ is singlino-like; for the corresponding cMSSM points, $\chi_{1}^{0}$ is mostly bino-like.) 


\begin{tabular}{|c|c|c|}
\hline Point: & cNMSSM.1 & cNMSSM.2 \\
\hline$M_{1 / 2}$ & 520 & 600 \\
\hline$m_{0}$ & 0 & 0 \\
\hline$A_{0}$ & -146.5 & -171 \\
\hline $\tan \beta$ & 22.2 & 23.3 \\
\hline$\lambda$ & $10^{-3}$ & $10^{-3}$ \\
\hline$\kappa$ & $1.1 \cdot 10^{-4}$ & $1.1 \cdot 10^{-4}$ \\
\hline$m_{\tilde{g}}$ & 1190 & 1360 \\
\hline$\left\langle m_{s q}\right\rangle$ & 1060 & 1200 \\
\hline$M_{\chi_{1}^{0}}$ & 146.4 & 171 \\
\hline$M_{\tilde{\tau}_{1}}$ & 150.5 & 174.5 \\
\hline$M_{H_{\mathrm{SM}}}$ & 114 & 115 \\
\hline$M_{H_{S}}$ & 103 & 121 \\
\hline$M_{A_{S}}$ & 179 & 209 \\
\hline \hline & $\mathrm{cMSSM} .1$ & $\mathrm{cMSSM} .2$ \\
\hline$m_{0}$ & 170 & 194 \\
\hline$M_{\chi_{1}^{0}}$ & 214.8 & 249.8 \\
\hline$M_{\tilde{\tau}_{1}}$ & 221.6 & 254.1 \\
\hline$\sigma_{\mathrm{Tot}}$ & $73 \mathrm{fb}$ & $28 \mathrm{fb}$ \\
\hline
\end{tabular}

Table 2. Parameters, some sparticle and Higgs masses, and the total sparticle production cross section for two cNMSSM benchmark points and nearby cMSSM points in the stau co-annihilation region, all of which account for a good dark matter relic density.

\section{Monte Carlo simulations and search channels}

For the calculation of the matrix elements we use MadGraph/MadEvent 5 [35], which includes Pythia 6.4 [36] for showering and hadronisation. Matching of the differential jet cross sections is performed according to the prescriptions in [37]. The sparticle branching ratios are obtained with the help of the code NMSDECAY [38] (based on SDECAY [39]), and are passed to Pythia.

The output is given in StdHEP-format to the fast detector simulation Delphes [40]. Inside Delphes, the appropriate ATLAS or CMS detector cards are used, together with the appropriate jet reconstruction algorithm. The jet reconstruction is performed with FastJet [41].

Subsequently we apply cuts corresponding to the following search channels S1 - S4:

- S1: ATLAS jets + missing transverse momentum (0 leptons) analysis [2]. 5 different signal regions are defined in table 1 in [2]. The cuts on $E_{T}^{\text {miss }}$ and $p_{T}$ of the leading jet are always $>130 \mathrm{GeV}$. 4 signal regions will be denoted by $2 j, 3 j, 4 j$ and $4 j L$, where the $p_{T}$ of the second, third and fourth jet satisfy always $p_{T}>40 \mathrm{GeV}$, and $m_{\mathrm{eff}}>1000 \mathrm{GeV}$ for $2 j, 3 j, 4 j$, but $m_{\mathrm{eff}}>500 \mathrm{GeV}$ for $4 j L$. In a fifth "high mass" region, denoted by $4 j H$, the second, third and fourth jet satisfy always $p_{T}>80 \mathrm{GeV}$, and $m_{\text {eff }}>1100 \mathrm{GeV}$ (see [2] for more details). 
- S2: ATLAS multijet + missing transverse momentum (0 leptons) analysis [4]. Here 4 signal regions are denoted by $7 j 55,8 j 55,6 j 80$ and $7 j 80$, where the numbers after the jet multiplicities denote the lower cut on the jet $p_{T}$ (see [4] for more details on the event selection).

- S3: CMS analysis of multilepton signatures [17]. Numerous different search channels have been considered in [17], depending on the lepton number, lepton species and charges. In addition, either a cut on $E_{T}^{\text {miss }}(\mathrm{MET})>50 \mathrm{GeV}$, or a cut on $H_{T}>$ $200 \mathrm{GeV}$ was applied $\left(H_{T}\right.$ is defined as the scalar sum of the transverse jet energies for all jets with $E_{T}>40 \mathrm{GeV}$ ). In some channels, excesses of events w.r.t. the SM have been observed in [17], but the event rates are still too small (and the systematical/statistical errors too large) to consider these excesses as significant.

Subsequently we sum over all leptons including (hadronically decaying) $\tau$ leptons, and distinguish only the search channels MET3, MET4, HT3 and HT4, where the numbers after MET or HT denote the number of leptons including $\tau$ 's. These search channels correspond to the lines $\sum l(l / \tau)(l / \tau)$ and $\sum l l(l / \tau)(l / \tau)$ in tables 1 and 2 in [17], where more details on the event selection can be found.

- S4: $2 \tau$ search by the CMS collaboration [12], where three signal regions have been defined. The first two require the presence of a lepton and a hadronically decaying $\tau\left(\tau_{h}\right)$ of opposite charge and $p_{T}>20 \mathrm{GeV}$, and two jets with $p_{T}>30 \mathrm{GeV}$. In the first signal region denoted as $e / \mu \tau_{h}$ high $E_{T}^{\text {miss }}$, one requires $E_{T}^{\text {miss }}>200 \mathrm{GeV}$ and $H_{T}>300 \mathrm{GeV}$. In the second signal region denoted as $e / \mu \tau_{h}$ high $H_{T}$, one requires $H_{T}>400 \mathrm{GeV}$ and $E_{T}^{\text {miss }}>150 \mathrm{GeV}$. In a third signal region denoted as $\tau_{h} \tau_{h}$, two hadronically decaying taus with $p_{T}>15 \mathrm{GeV}$, two jets with $p_{T}>100 \mathrm{GeV}$ and $H_{T}>200 \mathrm{GeV}$ are required. $\left(H_{T}\right.$ is defined as $H_{T}=\left|\sum_{i} \vec{p}_{T}^{i}\right|$, where the sum runs over all jets with $p_{T}>30 \mathrm{GeV}$.) This search channel is of relevance only for the cNMSSM.

In all cases we compared our event rates to those of the MSSM benchmark points given in these publications $[2,4,12,17]$, and found agreement within $20 \%-30 \%$. For each of the $\sim 80$ points studied in section 4 , we simulated at least $10^{4}$ events.

Clearly, our estimates of the efficiencies in the various channels are not as precise as the ones performed by the ATLAS and CMS collaborations. Hence, in the cases S1 and S2 we analyse ratios of efficiencies of benchmark lines 10.1.1, 10.4.1 and 40.2.1 of the sNMSSM to the corresponding benchmark points in the cMSSM with the same sparticle spectrum (except for the singlino-like LSP, see section 2). We can expect that systematic errors in our estimate of the efficiencies in the various channels cancel to a large extent in such ratios. Moreover, this procedure allows to translate bounds on $M_{1 / 2}$ obtained within the cMSSM into the parameter space of the NMSSM with a singlino-like LSP, as we will discuss in section 4 .

This strategy fails for the multilepton analysis S3 (and the $2 \tau$ analysis S4) where, in spite of the sum over different lepton species and charges, the efficiencies in the cMSSM are so small that they are of the order of our statistical errors. Therefore it is not appropriate to define sNMSSM/cMSSM ratios, and we will give estimates of the signal cross sections $\sigma$ after the event selection S3 for values of $\Delta_{M}$ which correspond to the largest efficiencies. These allow to estimate the signal rates for present and future luminosities. 


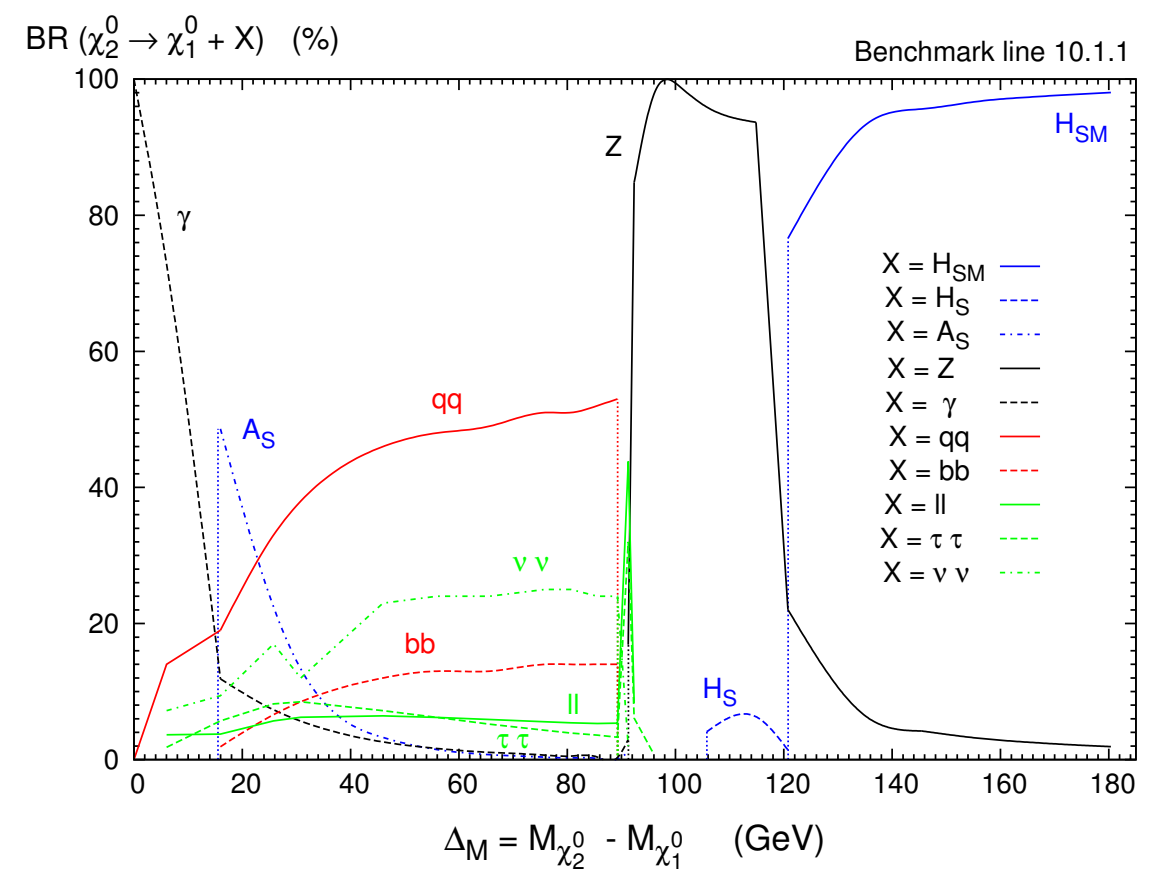

Figure 1. Branching fractions into the various states $X$ in the decay $\chi_{2}^{0} \rightarrow \chi_{1}^{0}+X$ as function of $\Delta_{M}$ for the benchmark line 10.1.1.

\section{Results}

In this section we show our results for the ratios of efficiencies $R=$ sNMSSM/cMSSM concerning the search channels S1 and S2, for the three benchmark lines 10.1.1, 10.4.1 and 40.2.1, as function of the bino-singlino mass difference, $\Delta_{M}$. In each case we first discuss the branching ratios for the decay $\chi_{2}^{0} \rightarrow \chi_{1}^{0}+X$ as function of $\Delta_{M}$, which are astonishingly complex since many different final states contribute for different values of $\Delta_{M}$. For the values of $\Delta_{M}$ where the branching ratios into leptons are large, we give estimates of the signal cross sections in the multilepton channels S3. Finally we provide the same information for the cNMSSM points cNMSSM.1 and cNMSSM.2, where we add estimates of the signal cross sections in the $2 \tau$ channels S4.

\subsection{Benchmark line 10.1.1}

The branching ratios for the decay $\chi_{2}^{0} \rightarrow \chi_{1}^{0}+X$ as function of $\Delta_{M}$ for the benchmark line 10.1.1 (for NMSSM-like parameters varying as given in table 1 ) is shown in figure 1.

As stated before, many different 2-body and 3-body final states ( $X$ corresponding to 1body or 2-body states, respectively) are possible. For a small bino-singlino mass difference $\Delta_{M}$, the dominant $\chi_{2}^{0}$ decay mode is the radiatively induced 2-body decay $\chi_{2}^{0} \rightarrow \chi_{1}^{0}+\gamma$. However, the $\gamma$ energy would probably be too small to allow its detection. For larger $\Delta_{M}$ in the range $\sim 20-25 \mathrm{GeV}$, the 2-body decay into the mostly singlet-like CP-odd Higgs boson $A_{S}$ dominates. The possible relevance of $A_{S}$ production (and/or $H_{S}$ production) in neutralino decays in the NMSSM has already been underlined in $[42,43]$. In turn, $A_{S}$ will 


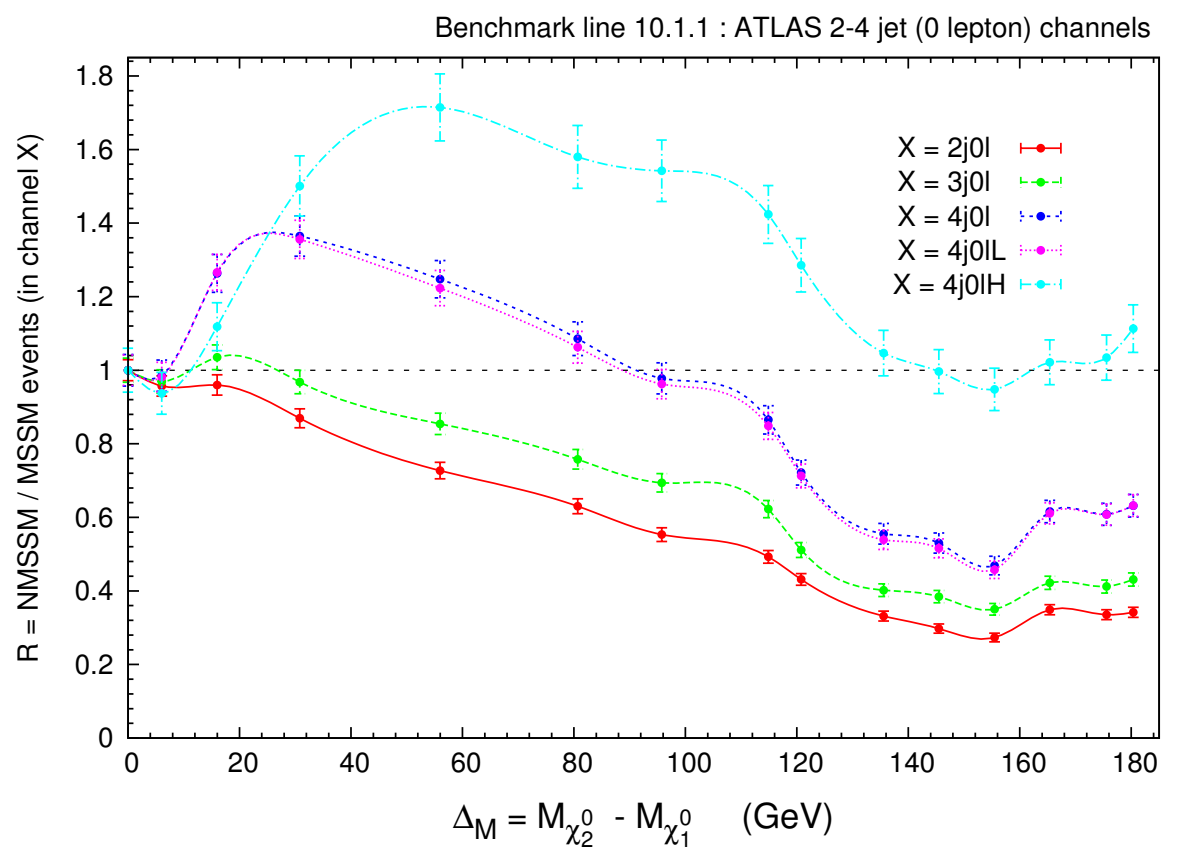

Figure 2. The ratios $R$ of efficiencies as function of $\Delta_{M}$ in the sNMSSM w.r.t. the cMSSM point 10.1.1 in 5 different signal regions of the ATLAS jets + missing transverse momentum analysis.

decay dominantly into a pair of $b$-quarks. For $\Delta_{M}<M_{Z}$ one finds a plethora of 3-body decays into $q \bar{q}, b \bar{b}$, leptons (electrons and muons), $\tau^{+} \tau^{-}$and neutrinos most of which are mediated by the $Z$ boson and $A_{S}$. For $M_{Z}<\Delta_{M}<M_{H_{\mathrm{SM}}}, \chi_{2}^{0}$ decays nearly exclusively into $\chi_{2}^{0} \rightarrow \chi_{1}^{0}+Z$ (with a small branching fraction into $H_{S}$ ), and for $\Delta_{M}>M_{H_{\mathrm{SM}}}$ nearly exclusively into the SM-like Higgs boson.

Next we apply the event selections and cuts according to the 5 different signal regions of the ATLAS jets + missing transverse momentum (0 leptons) analysis in [2] for various values of $\Delta_{M}$ (see the search channels S1 in section 3). The same cuts are applied to the cMSSM point 10.1.1, and subsequently we determine the ratios $R$ of efficiencies sNMSSM/cMSSM. The results for $R$ are shown in figure 2. (Here and in the following figures for $R$, the error bars indicate statistical errors only, which follow from the fact that we have simulated about 10000 events per point.)

We see that i) $R$ is nearly always larger than 1 for the $4 j H$ signal region; ii) for the $4 j$ and $4 j L$ signal regions, $R>1$ for $\Delta_{M} \lesssim 90 \mathrm{GeV}$, but $R<1$ for $\Delta_{M} \gtrsim 100 \mathrm{GeV}$, and iii) $R<1$ everywhere for the $3 j$ and $2 j$ signal regions. Notably for the latter, $R$ can drop to $\sim 0.3$ for $\Delta_{M} \sim 150 \mathrm{GeV}$. For $\Delta_{M}>115 \mathrm{GeV}$, the dominant $\chi_{2}^{0}$ decay is $\chi_{2}^{0} \rightarrow \chi_{1}^{0}+H_{\mathrm{SM}}$ and, whereas $H_{\mathrm{SM}}$ carries away a considerable amount of (no longer invisible) transverse energy, its decay products ( $b$-jets) contribute to the signatures. Hence, the reduction of $R$ is dominant for the $3 j$ and $2 j$ signal regions, which hardly profit from the additional $b$-jets.

The impact of the additional jets is also clearly visible in the case of the ATLAS multijet + missing transverse momentum (0 leptons) analysis in [4] (see the search channels S2 in section 3 ). We proceed as above, and study the ratios $R$ of efficiencies in the sNMSSM 


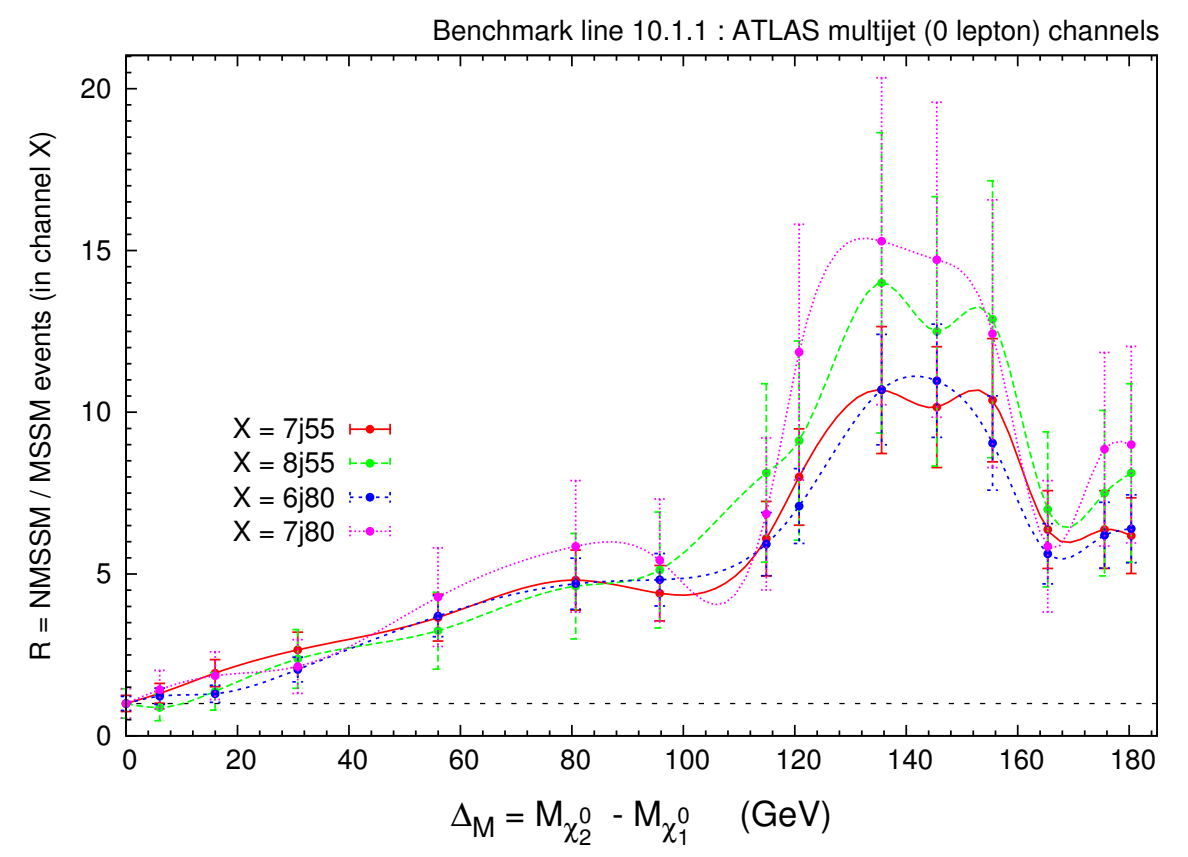

Figure 3. The ratios $R$ of efficiencies as function of $\Delta_{M}$ in the sNMSSM w.r.t. the cMSSM point 10.1.1 in 4 different signal regions of the ATLAS multijet + missing transverse momentum analysis.

w.r.t. the cMSSM in the 4 different signal regions as function of $\Delta_{M}$. The results are shown in figure 3.

Due to the additional jets in the final states and the somewhat weaker cut on $E_{T}^{\text {miss }}$ in [4], $R$ can become considerably larger than 1 , notably for $\Delta_{M} \gtrsim 115 \mathrm{GeV}$ where the $b$-jets from $H_{\mathrm{SM}}$ contribute to the signal region.

Given the present absence of clear signals for supersymmetry, an important question is to which extent the modified efficiencies in the sNMSSM affect the present lower boundaries in the $m_{0}-M_{1 / 2}$ plane. The answer is not obvious since, depending on the search channel, the efficiencies in the sNMSSM can be larger or smaller than in the cMSSM. Hence we have to compare the search channels which are actually relevant for the most stringent bounds in the $m_{0}-M_{1 / 2}$ plane.

The cMSSM point 10.1.1 is part of the benchmark line 10.1 in [24], which is specified by relatively low values for $m_{0}$ and $A_{0}=0, \tan \beta=10$. For the same values of $A_{0}$ and $\tan \beta$, the boundaries in the $m_{0}-M_{1 / 2}$ plane implied by the ATLAS jets + missing transverse momentum analysis in [2] can be found, channel by channel, on the web page [25]. One finds that, for these low values of $m_{0}$, the constraints are dominated by the $2 j / 3 j$ signal regions implying $M_{1 / 2} \gtrsim 470 / 450 \mathrm{GeV}$, respectively. The $2 j / 3 j$ signal regions are precisely those for which the modified efficiencies in the sNMSSM can be considerably lower (by a factor $\sim 1 / 3)$ than in the cMSSM, as can be seen in figure 2 for $\Delta_{M} \sim 150 \mathrm{GeV}$.

Hence, the constraints from the absence of excesses in the $2 j / 3 j$ signal regions can accommodate, in the sNMSSM with $\Delta_{M} \sim 150 \mathrm{GeV}$, a production cross section which is about 3 times larger than in the cMSSM, corresponding to a somewhat smaller 


\begin{tabular}{|c|c|c|c|c|}
\hline Channel: & MET3 & MET4 & HT3 & HT4 \\
\hline$\sigma[\mathrm{fb}]$ & $1.61 \pm 0.09$ & $0.29 \pm 0.04$ & $1.63 \pm 0.09$ & $0.31 \pm 0.04$ \\
\hline
\end{tabular}

Table 3. Signal cross sections for the CMS multilepton search channels MET3, MET4, HT3 and HT4 (see text) for the sNMSSM with parameters corresponding to the cMSSM point 10.1.1, and a light singlino-like LSP with $M_{\chi_{1}^{0}} \sim 25 \mathrm{GeV}$.

value for $M_{1 / 2}$. Using again the next-to-leading order production cross sections from Prospino $[32,33]$, we find that the latter decrease with increasing $M_{1 / 2}$ (implying increasing squark and gluino masses) roughly like $M_{1 / 2}^{-8.5}$. Thus in the sNMSSM for $\Delta_{M} \sim 150 \mathrm{GeV}$, the lower bound on $M_{1 / 2}$ from the absence of excesses in the $2 j / 3 j$ signal region is lower than in the cMSSM by a factor $(1 / 3)^{1 / 8.5} \sim 0.88$, leading to $M_{1 / 2} \gtrsim 415 \mathrm{GeV}$ instead of $M_{1 / 2} \gtrsim 470 \mathrm{GeV}$ for $m_{0} \sim 125 \mathrm{GeV}, A_{0}=0$ and $\tan \beta=10$.

Of course we must verify whether such a reduced value of $M_{1 / 2}$ is consistent with constraints from the other search channels, notably those in which the efficiencies in the sNMSSM are larger than in the cMSSM: First, in the remaining search channels in the ATLAS jets + missing transverse momentum analysis in [2] the efficiencies in the sNMSSM with $\Delta_{M} \sim 150 \mathrm{GeV}$ are never enhanced, and $M_{1 / 2} \gtrsim 415 \mathrm{GeV}$ remains consistent with the corresponding bounds. Also, the lower bounds on $M_{1 / 2}$ from the ATLAS multijet + missing transverse momentum analysis in [4], for $m_{0} \sim 125 \mathrm{GeV}$, are so low that the enhanced efficiencies in the sNMSSM from figure 3 remain consistent with present bounds for $M_{1 / 2} \gtrsim 415 \mathrm{GeV}$.

We have all reasons to expect that these conclusions - a reduced lower bound on $M_{1 / 2}$ by a factor $\sim 0.88$ in the sNMSSM with $\Delta_{M} \sim 150 \mathrm{GeV}$ w.r.t. the cMSSM for corresponding values of $m_{0}, A_{0}$ and $\tan \beta$ - remain valid in the light of the constraints from CMS in [9], which are slightly stronger $\left(M_{1 / 2} \gtrsim 540 \mathrm{GeV}\right.$, to be replaced by $\left.M_{1 / 2} \gtrsim 475 \mathrm{GeV}\right)$ : Again, the constraints are dominated by the $2 / 3$ jet analyses (see [8]), for which the efficiencies in the sNMSSM can be reduced due to less missing transverse momentum, thus being compatible with a larger production cross section than in the cMSSM. (A detailed analysis of all available SUSY search channels would go beyond the scope of the present paper.)

However, it is of interest to verify to which extent these sNMSSM scenarios would contribute to the CMS analysis of multilepton signatures in [17] (see the search channels S3 in section 3). The largest efficiencies in the sNMSSM are found in the region $\Delta_{M} \sim 180 \mathrm{GeV}$, corresponding to $M_{\chi_{1}^{0}} \sim 25 \mathrm{GeV}$; the corresponding signal cross sections (including statistical errors) are given in table 3 .

We see that the values for $\sigma$ are small and would hardly give visible event rates for luminosities of a few $\mathrm{fb}^{-1}$. Hence sNMSSM scenarios with somewhat lower values of $M_{1 / 2}$ as compared to cMSSM scenarios, as allowed by the $2 j / 3 j$ search channels, are not ruled out. For the corresponding cMSSM point 10.1.1, leptons are expected from the cascade decay of the wino-like NLSP $\chi_{2}^{0} \rightarrow \chi_{1}^{0}+\bar{l} l / \bar{\tau} \tau$ (see the discussion in [24]). Still, the corresponding signal cross sections in these channels are smaller by about a factor $1 / 6$ (for MET3/4) or $1 / 3$ (for HT3/4); in the future, such differences can help distinguishing different scenarios for supersymmetry. 


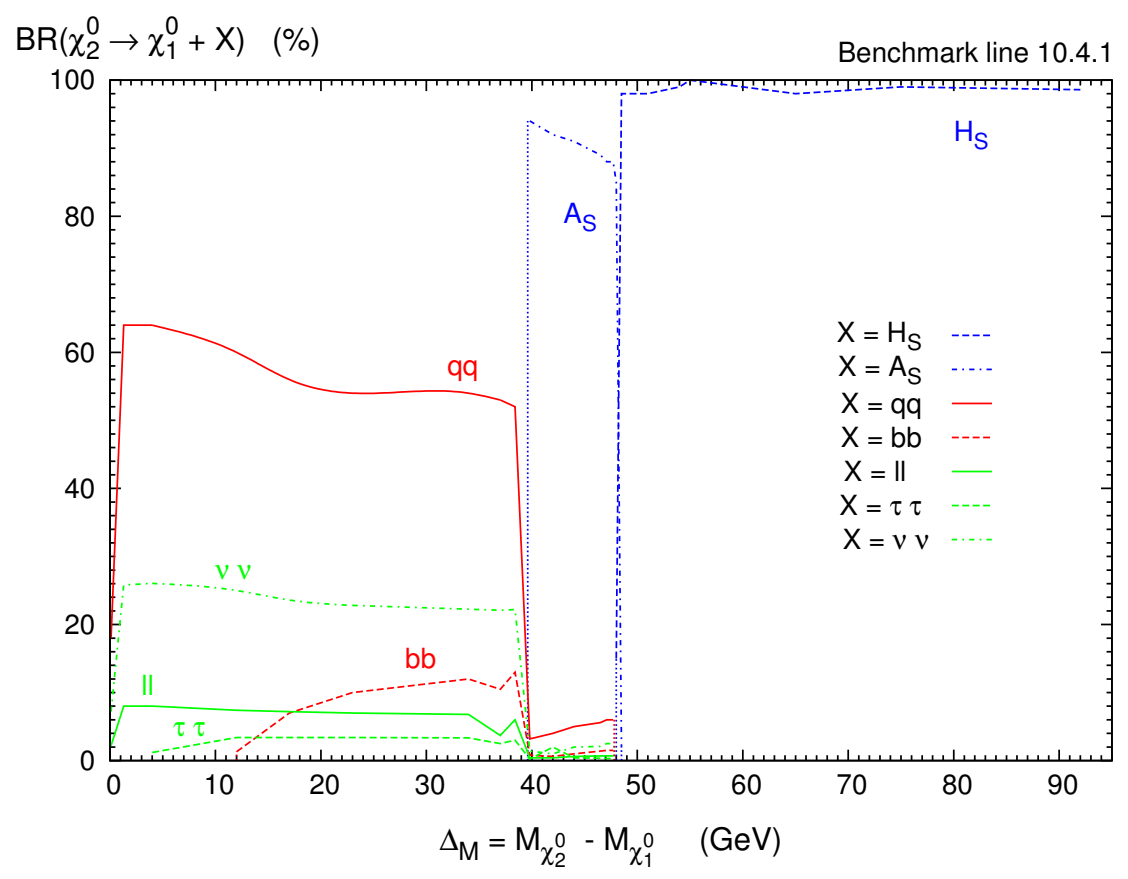

Figure 4. Branching fractions into the various states $X$ in the decay $\chi_{2}^{0} \rightarrow \chi_{1}^{0}+X$ as function of $\Delta_{M}$ for the benchmark line 10.4.1.

\subsection{Benchmark line 10.4.1}

Compared to the sNMSSM benchmark line 10.1.1, the benchmark line 10.4.1 corresponds to a larger value of $\lambda=0.013$ and $m_{0}=750 \mathrm{GeV}$, but a smaller value for $M_{1 / 2}=350 \mathrm{GeV}$, as given in table 1. Due to the larger mixings in the Higgs sector for $\lambda=0.013$, it is more difficult to satisfy LEP constraints on light Higgs bosons. Hence the singlino-like neutralino mass has to be larger than $\sim 50 \mathrm{GeV}$, or $\Delta_{M} \lesssim 93 \mathrm{GeV}$. The branching fractions into the various states $X$ in the decay $\chi_{2}^{0} \rightarrow \chi_{1}^{0}+X$ are shown in figure 4 .

In contrast to figure 1 , the radiatively induced 2-body decay $\chi_{2}^{0} \rightarrow \chi_{1}^{0}+\gamma$ is no longer dominant for a small bino-singlino mass difference $\Delta_{M}$. However, for $35 \mathrm{GeV} \lesssim \Delta_{M}$ $\lesssim 45 \mathrm{GeV}$, the 2-body decay into the mostly singlet-like CP-odd Higgs boson $A_{S}$ dominates again. Due to the larger value of $\lambda$ compared to the corresponding analysis of the 10.1.1 line (see figure 1), the 2-body decay into the mostly singlet-like $H_{S}$ dominates for $48 \mathrm{GeV} \lesssim \Delta_{M}$.

Next we consider again the ratios $R$ of efficiencies in the SNMSSM as a function of $\Delta_{M}$ w.r.t. the cMSSM, for the ATLAS jets + missing transverse momentum analysis in [2] (search channels S1) and the ATLAS multijet + missing transverse momentum analysis in [4] (search channels S2). The results are shown in figure 5.

In the case of the ATLAS jets + missing transverse momentum analysis - the upper panel of figure 5 - we see that now $R$ can decrease to $\sim 0.3$ for all search channels for $\Delta_{M} \sim 75 \mathrm{GeV}\left(M_{\chi_{1}^{0}} \sim 70 \mathrm{GeV}\right)$, becoming as small as $R \sim 0.2$ for the most relevant $2 j / 3 j$ channels. The increase of $R$ for the ATLAS multijet + missing transverse momentum 

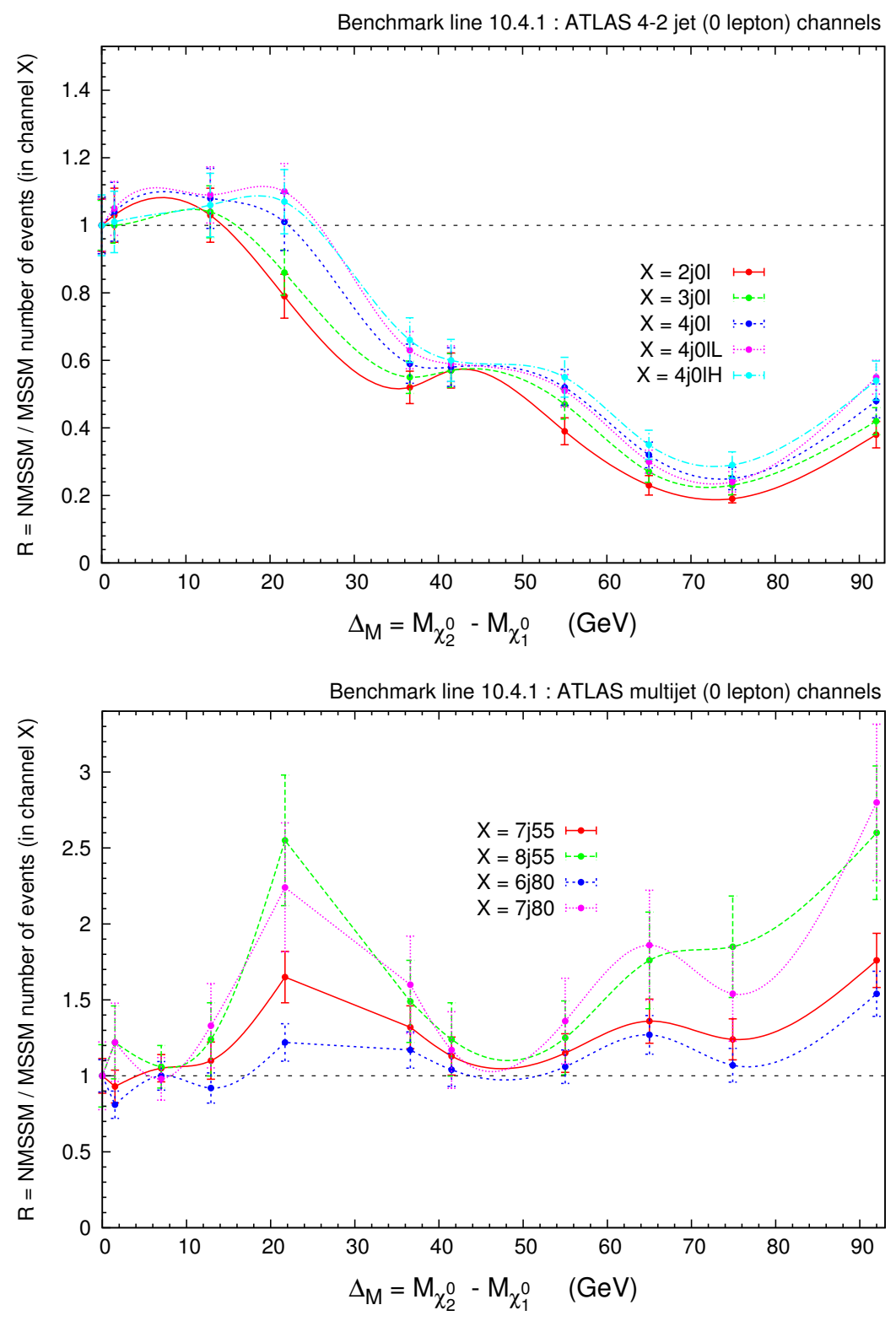

Figure 5. The ratios $R$ of efficiencies as function of $\Delta_{M}$ in the sNMSSM w.r.t. the cMSSM point 10.4.1 in 5 different signal regions of the ATLAS jets + missing transverse momentum analysis (upper panel) and in 4 different signal regions of the ATLAS multijet + missing transverse momentum analysis (lower panel).

analysis shown in the lower panel of figure 5 is less pronounced than in figure 3 . As explained in [24], the reason is that here the gluino is lighter than the squarks and its dominant 3-body decays yield higher fractions of final states with more hadronic jets, already in the cMSSM. Hence the relative increase of multijet efficiencies in the sNMSSM is smaller than for the previous line 10.1.1.

As before, we can estimate to what extent the reduced value of $R \sim 0.2$ for the most relevant $2 j / 3 j$ channels alleviates the lower bound on $M_{1 / 2}$ : For $m_{0}=750 \mathrm{GeV}$, the 


\begin{tabular}{|c|c|c|c|c|}
\hline Channel: & MET3 & MET4 & HT3 & HT4 \\
\hline$\sigma[\mathrm{fb}]$ & $2.6 \pm 0.2$ & $\lesssim 0.1$ & $1.5 \pm 0.1$ & $\lesssim 0.1$ \\
\hline
\end{tabular}

Table 4. Signal cross sections for the CMS multilepton search channels for the sNMSSM with parameters corresponding to the cMSSM point 10.4.1, and a singlino-like LSP with $M_{\chi_{1}^{0}} \sim 100 \mathrm{GeV}$.

production cross sections for squarks/gluinos decrease roughly like $M_{1 / 2}^{-6.3}$. Thus in the sNMSSM for $\Delta_{M} \sim 75 \mathrm{GeV}$, the lower bound on $M_{1 / 2}$ from the absence of excesses in the $2 j / 3 j$ signal region is lower than in the cMSSM by a factor $(1 / 5)^{1 / 6.3} \sim 0.75$. Again this conclusion is not affected by the larger efficiencies in the less sensitive multijet channels.

The largest efficiencies in the sNMSSM in the multilepton channels MET3/HT3 analysed by CMS in [17] are found in the region $\Delta_{M} \sim 45 \mathrm{GeV}$ corresponding to $M_{\chi_{1}^{0}} \sim$ $100 \mathrm{GeV}$, and originate from tau leptons arising from $A_{S}$ decays (see figure 4 ); the associated signal cross sections are given in table 4. (The signal cross sections in the MET4/HT4 channels, requiring 4 leptons passing the cuts, are even smaller than for the 10.1.1 point shown in table 3 , and are of the order of our statistical errors.)

We observed that events originating from squark/gluino production contribute simultaneously to the MET and HT search channels, and would give practically identical signal cross sections. However, events originating from neutralino/chargino/slepton production only contribute to the MET search channels, since they lead to less jets which would be required for the HT cuts; this explains the slightly larger signal cross section in MET3 compared to HT3. In the cMSSM, the signal cross sections are smaller by about a factor $1 / 3$, but even in the NMSSM the event rates are obviously small for luminosities of a few $\mathrm{fb}^{-1}$.

\subsection{Benchmark line 40.2.1}

Now we study a region in parameter space with larger values of $\tan \beta=40$ and $A_{0}=$ $-500 \mathrm{GeV}$, as defined in table 1 . The branching fractions into the various states $X$ in the decay $\chi_{2}^{0} \rightarrow \chi_{1}^{0}+X$ are shown in figure 6 . In this case, the 3 -body decays of $\chi_{2}^{0}$ into leptons, taus and quark pairs dominate for $\Delta_{M} \lesssim 90 \mathrm{GeV}$. For $90 \mathrm{GeV} \lesssim \Delta_{M} \lesssim 115 \mathrm{GeV}$, 2-body decays into $H_{S}$ (and $Z$ ) dominate whereas, as before, 2-body decays into $H_{\mathrm{SM}}$ dominate for $\Delta_{M} \gtrsim 115 \mathrm{GeV}$.

For the search channels $\mathrm{S} 1$ and $\mathrm{S} 2$, the results for the ratios $R$ of efficiencies in the sNMSSM as function of $\Delta_{M}$ w.r.t. the cMSSM are shown in figure 7 .

Here the decrease of $R$ for the ATLAS jets + missing transverse momentum analysis is even stronger than in the previous cases; for the most relevant $2 j$ and $3 j$ channels, $R$ drops below 0.15 for $\Delta_{M} \gtrsim 130 \mathrm{GeV}\left(M_{\chi_{1}^{0}} \lesssim 60 \mathrm{GeV}\right)$. For the ATLAS multijet + missing transverse momentum analysis $R$ increases up to $\sim 5$ in this region of $\Delta_{M}$, which is again less pronounced than in the 10.1.1 analysis of figure 3. The reason is that already in the cMSSM the gluino decays dominantly into a stop+top pair [24], yielding again (but for a different reason) higher fractions of final states with more hadronic jets making the relative increase of multijet efficiencies in the sNMSSM smaller than for the point 10.1.1. 


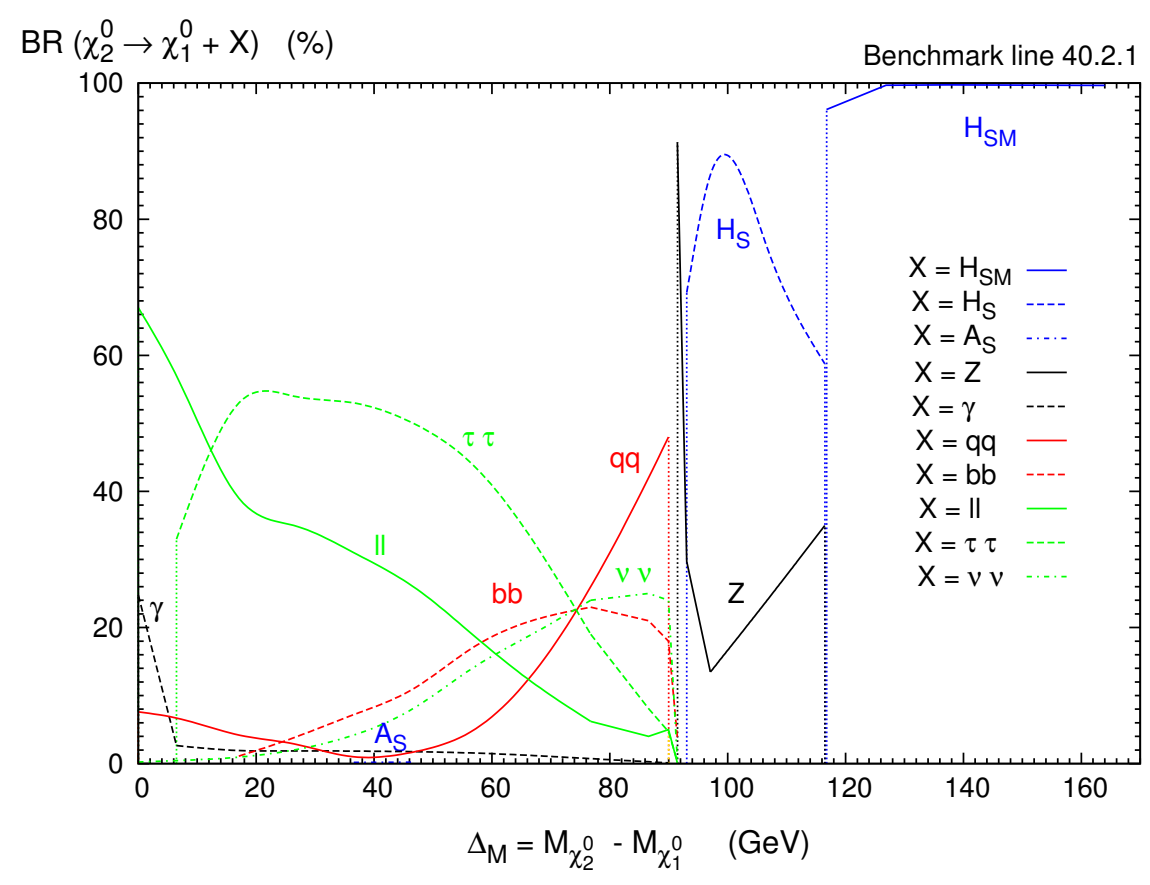

Figure 6. Branching fractions into the various states $X$ in the decay $\chi_{2}^{0} \rightarrow \chi_{1}^{0}+X$ as function of $\Delta_{M}$ for the benchmark line 40.2.1.

\begin{tabular}{|c|c|c|c|c|}
\hline Channel: & MET3 & MET4 & HT3 & HT4 \\
\hline$\sigma[\mathrm{fb}]$ & $2.72 \pm 0.03$ & $0.11 \pm 0.01$ & $2.72 \pm 0.03$ & $0.11 \pm 0.01$ \\
\hline
\end{tabular}

Table 5. Signal cross sections for the CMS multilepton search channels for the sNMSSM with parameters corresponding to the cMSSM point 40.2.1, and a light singlino-like LSP with $M_{\chi_{1}^{0}} \sim$ $40 \mathrm{GeV}$.

Concerning the multilepton channels analysed by CMS, the largest efficiencies in the sNMSSM are found in the region $\Delta_{M} \sim 145 \mathrm{GeV}$ corresponding to $M_{\chi_{1}^{0}} \sim 40 \mathrm{GeV}$; the corresponding signal cross sections are given in table 5 .

For the cMSSM point 40.2.1, these signal cross sections are smaller by a factor less than $1 / 10$. The origin of the larger multilepton signal cross section in the sNMSSM is the 3-body decay of $\chi_{2}^{0}$ into $\chi_{1}^{0}$ plus leptons, as shown in figure 6 (see also $[44,45]$ ), with leptons sufficiently energetic to survive the cuts in the CMS analysis.

Since in the sNMSSM the reduction of $R$ down to 0.15 in the most relevant $2 j / 3 j$ channels is stronger than before, the sNMSSM in this region of parameter space could be compatible, in the absence of signals, with sparticle production cross sections about 7 times larger than in the cMSSM. Since here (for $m_{0}=550 \mathrm{GeV}, A_{0}=-500 \mathrm{GeV}, \tan \beta=40$ ) the squark/gluino production cross section decreases $\sim M_{1 / 2}^{-7.5}$, for $\Delta_{M} \gtrsim 130 \mathrm{GeV}$ the cNMSSM is compatible with values of $M_{1 / 2}$ which are smaller than in the cMSSM by about 0.75. (Again this conclusion is not affected by the enhancement of $R$ in the less sensitive multijet or multilepton channels.) These potential attenuations of lower bounds on $M_{1 / 2}$ are not dramatic, but neither completely negligible. 

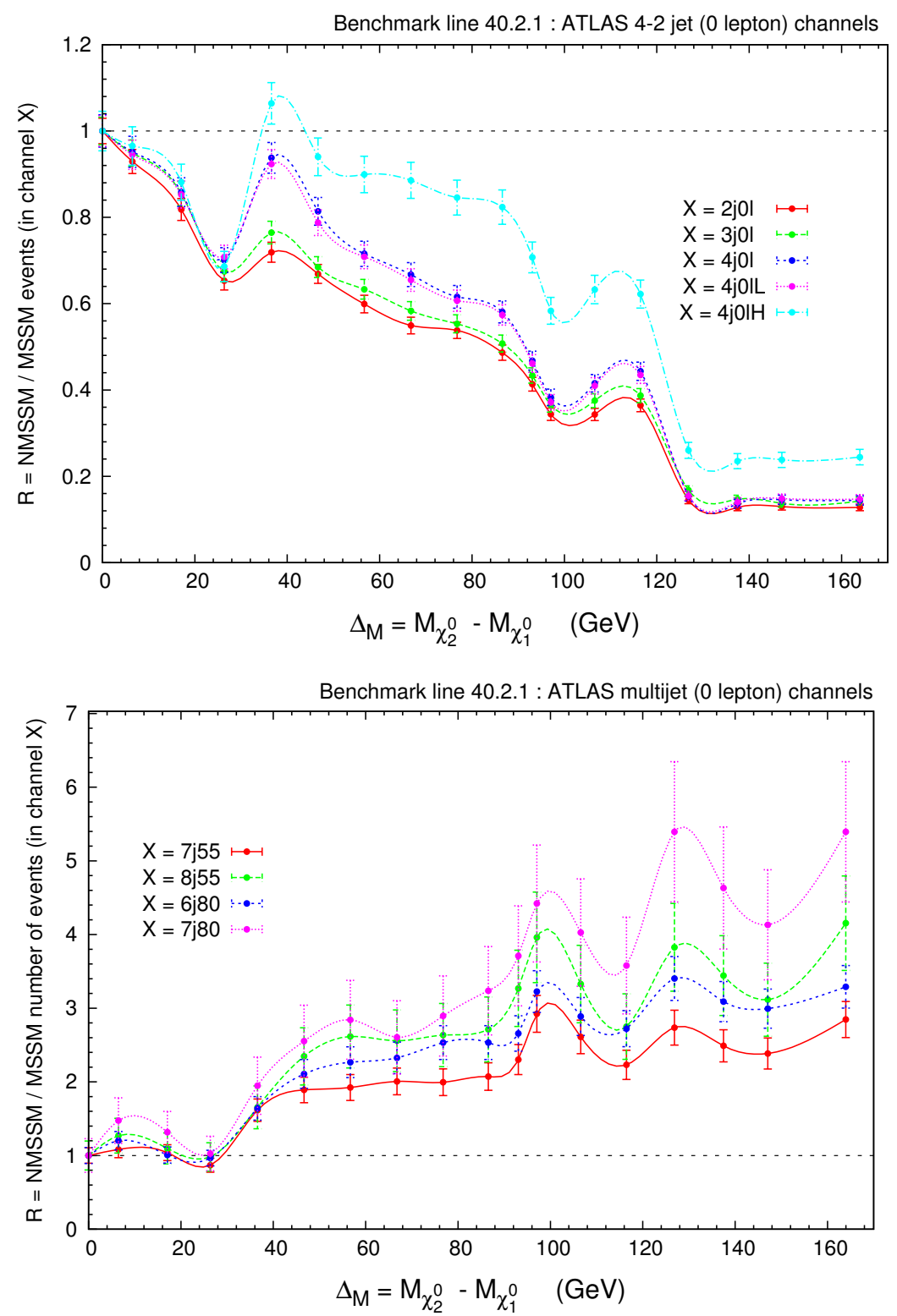

Figure 7. The ratios $R$ of efficiencies as function of $\Delta_{M}$ in the sNMSSM w.r.t. the cMSSM point 40.2.1 in 5 different signal regions of the ATLAS jets + missing transverse momentum analysis (upper panel) and in 4 different signal regions of the ATLAS multijet + missing transverse momentum analysis (lower panel).

\subsection{The cNMSSM}

The parameters and some sparticle and Higgs masses for two cNMSSM benchmark points, as well as nearby cMSSM points in the stau co-annihilation region, have been given in table 2 . In this case the $\tilde{\tau}_{1}$ is the NLSP, decaying as $\tilde{\tau}_{1} \rightarrow \tau+\chi_{1}^{0}$, where $\chi_{1}^{0}$ is mostly singlinolike in the cNMSSM, but mostly bino-like for the cMSSM points. Due to the small $\tilde{\tau}_{1}-\chi_{1}^{0}$ mass difference, these $\tau$ leptons are however quite soft. Harder $\tau$ leptons appear in the sparticle decays into $\tilde{\tau}_{1}$. One such sparticle decay appears in every sparticle decay cascade in the cNMSSM, but only occasionally in the cMSSM in the stau co-annihilation region. 


\begin{tabular}{|c|c|c|c|c|c|}
\hline Channel: & $2 j$ & $3 j$ & $4 j$ & $4 j L$ & $4 j H$ \\
\hline$R_{1}$ & $0.63 \pm 0.04$ & $0.73 \pm 0.05$ & $0.86 \pm 0.06$ & $0.86 \pm 0.06$ & $0.95 \pm 0.08$ \\
\hline$R_{2}$ & $0.65 \pm 0.03$ & $0.75 \pm 0.03$ & $0.95 \pm 0.06$ & $0.96 \pm 0.06$ & $1.1 \pm 0.1$ \\
\hline
\end{tabular}

Table 6. Ratios $R_{1}=$ (cNMSSM.1/cMSSM.1) and $R_{2}=($ cNMSSM.2/cMSSM. 2$)$ of efficiencies for two points of the cNMSSM w.r.t. the cMSSM in 5 different signal regions of the ATLAS jets + missing transverse momentum analysis.

\begin{tabular}{|c|c|c|c|c|}
\hline Channel: & MET3 & MET4 & HT3 & HT4 \\
\hline$\sigma[\mathrm{fb}]$ (cNMSSM.1) & $4.4 \pm 0.2$ & $0.7 \pm 0.1$ & $2.7 \pm 0.1$ & $0.41 \pm 0.05$ \\
\hline$\sigma[\mathrm{fb}]$ (cNMSSM.2) & $1.63 \pm 0.06$ & $0.36 \pm 0.03$ & $0.94 \pm 0.04$ & $0.18 \pm 0.02$ \\
\hline
\end{tabular}

Table 7. Signal cross sections for the CMS multilepton search channels for two points of the cNMSSM.

\begin{tabular}{|c|c|c|c|}
\hline Channel: & $e / \mu \tau_{h}$ high $E_{T}^{\text {miss }}$ & $e / \mu \tau_{h}$ high $H_{T}$ & $\tau_{h} \tau_{h}$ \\
\hline$\sigma[\mathrm{fb}](\mathrm{cNMSSM} .1)$ & $2.2 \pm 0.1$ & $2.4 \pm 0.1$ & $0.65 \pm 0.05$ \\
\hline$\sigma[\mathrm{fb}](\mathrm{cNMSSM} .2)$ & $0.77 \pm 0.03$ & $0.81 \pm 0.03$ & $0.19 \pm 0.01$ \\
\hline
\end{tabular}

Table 8. Signal cross sections for the CMS $2 \tau$ search channels for two cNMSSM points.

First we compare, as before, the ratios $R$ of efficiencies in the cNMSSM w.r.t. the cMSSM in 5 different signal regions of the ATLAS jets + missing transverse momentum analysis (search channels $\mathrm{S} 1$ ); the results are given in table 6 .

With $R_{1,2} \sim 0.6-1.1$, the differences between the cNMSSM and the cMSSM are not spectacular in these search channels. In the case of the multijets + missing transverse momentum analysis (S2), efficiencies are so small that a comparison is not meaningful. In table 7 we give the signal cross sections for the multilepton search channels analysed by CMS (S3).

In spite of the smaller total sparticle production cross sections in the cNMSSM compared to the sNMSSM benchmark lines, the signal cross sections in the multilepton search channels are at least of the same order, notably for the MET channels, where no hard jets are required: Here neutralino/chargino/slepton/stau production processes contribute, in contrast to the HT channels which require hard jets originating from squark/gluino production. (For the corresponding cMSSM points cMSSM.1 and cMSSM.2, these signal cross sections are smaller by about a factor $1 / 20$ and not shown here.) In [17], $2.1 \mathrm{fb}^{-1}$ of luminosity have been analysed by CMS in the multilepton channels. No significant excesses are expected within the cNMSSM at present, but these search channels can become sensitive to the cNMSSM in the future.

Due to the large number of $\tau$ leptons in the final states, it becomes interesting to study the three signal regions of the CMS $2 \tau$ search channels (S4, see section 3 ) for the cNMSSM; the corresponding signal cross sections are shown in table 8 . 
(Again, for the corresponding cMSSM points cMSSM.1 and cMSSM.2 these signal cross sections are smaller by about a factor 1/20.) In [28] it was estimated that only the LHC at $14 \mathrm{TeV}$ c.m. energy would become sensitive to the cNMSSM. However, combining multilepton and $2 \tau$ search channels and increasing the integrated luminosity to $\sim 20 \mathrm{fb}^{-1}$, already the LHC with $7 \mathrm{TeV}$ c.m. energy could become sensitive to the low $M_{1 / 2}$ regime of the cNMSSM in the future.

\section{Conclusions and outlook}

In the NMSSM with a singlino-like LSP, it is easy to estimate qualitatively how the signatures for supersymmetry are modified with respect to the MSSM: The additional cascade $\chi_{2}^{0} \rightarrow \chi_{1}^{0}+X$ will reduce the missing energy, but will provide additional jets or leptons. In the present paper we studied this issue quantitatively for several benchmark points (lines in the sNMSSM), considering several supersymmetry search channels. For the considered benchmark lines, we found that the efficiencies can drop by a factor $\sim 1 / 3$ to $\sim 1 / 7$ with respect to the MSSM in the most relevant $2 / 3$ jet + missing energy search channels. This can reduce the present lower bounds on $M_{1 / 2}$ by a factor $\sim 0.9-0.75$ for parameter regions of the sNMSSM corresponding to large bino-singlino mass differences. The corresponding increase of efficiencies in multijet or multilepton search channels is not strong enough to affect this conclusion.

In addition, we studied the cNMSSM, in which the $\tilde{\tau}_{1}$ is the NLSP and the $\tilde{\tau}_{1}$-singlino mass difference is small (in order to comply with the WMAP bounds on the dark matter relic density), for the lowest possible values of $M_{1 / 2}$. cMSSM points with similar values of $m_{0}$ and $M_{1 / 2}$ are not excluded by present searches. Since the efficiencies in the most relevant $2 / 3$ jet + missing energy channels in the cNMSSM turn out to be similar, present searches are not sensitive to the cNMSSM either. However, in the future the signal cross sections in the multilepton and $2 \tau$ search channels could give hints for the cNMSSM at the LHC with $7 \mathrm{TeV}$ (or $8 \mathrm{TeV}$ ) c.m. energy for larger integrated luminosities.

Clearly, in a first study of this kind we could only "scratch the tip of the iceberg": First, many more search channels (different combinations of and cuts on missing transverse energy, jets and leptons) are studied by the ATLAS and CMS collaborations. Second, we did not cover even the sNMSSM parameter space completely; we confined ourselves to regions similar to cMSSM benchmark points with moderate (but not excluded) values of both $m_{0}$ and $M_{1 / 2}$. As in the MSSM, the parameter space of the general NMSSM is much larger, and studies similar to those within the general MSSM [19-22] could reveal more regions in the NMSSM to which the SUSY search channels are less sensitive than in the MSSM. Third, more refined studies of efficiencies in various search channels as function of the final states in the additional bino $\rightarrow$ singlino cascade can help to clarify under which circumstances the NMSSM can be distinguished from the MSSM independently from the Higgs sector. Hence the present study can and should be extended in many different ways. 


\section{Acknowledgments}

U. E. acknowledges support from the French ANR LFV-CPV-LHC.

Open Access. This article is distributed under the terms of the Creative Commons Attribution License which permits any use, distribution and reproduction in any medium, provided the original author(s) and source are credited.

\section{References}

[1] U. Ellwanger, C. Hugonie and A.M. Teixeira, The next-to-minimal supersymmetric standard model, Phys. Rept. 496 (2010) 1 [arXiv:0910.1785] [INSPIRE].

[2] ATLAS collaboration, G. Aad et al., Search for squarks and gluinos using final states with jets and missing transverse momentum with the ATLAS detector in $\sqrt{s}=7 \mathrm{TeV}$ proton-proton collisions, Phys. Lett. B 710 (2012) 67 [arXiv:1109.6572] [INSPIRE].

[3] ATLAS collaboration, G. Aad et al., Search for supersymmetry in final states with jets, missing transverse momentum and one isolated lepton in $\sqrt{s}=7 \mathrm{TeV}$ pp collisions using $1 \mathrm{fb}^{-1}$ of ATLAS data, Phys. Rev. D 85 (2012) 012006 [arXiv:1109.6606] [INSPIRE].

[4] ATLAS collaboration, G. Aad et al., Search for new phenomena in final states with large jet multiplicities and missing transverse momentum using $\sqrt{s}=7 \mathrm{TeV}$ pp collisions with the ATLAS detector, JHEP 11 (2011) 099 [arXiv:1110.2299] [INSPIRE].

[5] ATLAS collaboration, G. Aad et al., Searches for supersymmetry with the ATLAS detector using final states with two leptons and missing transverse momentum in $\sqrt{s}=7 \mathrm{TeV}$ proton-proton collisions, Phys. Lett. B 709 (2012) 137 [arXiv:1110.6189] [INSPIRE].

[6] ATLAS collaboration, G. Aad et al., Search for diphoton events with large missing transverse momentum in $1 \mathrm{fb}^{-1}$ of $7 \mathrm{TeV}$ proton-proton collision data with the ATLAS detector, arXiv:1111.4116 [INSPIRE].

[7] ATLAS collaboration, G. Aad et al., Search for scalar bottom pair production with the ATLAS detector in pp collisions at $\sqrt{s}=7 \mathrm{TeV}$, arXiv:1112.3832 [INSPIRE].

[8] CMS collaboration, V. Khachatryan et al., Search for supersymmetry in pp collisions at $7 \mathrm{TeV}$ in events with jets and missing transverse energy, Phys. Lett. B 698 (2011) 196 [arXiv:1101.1628] [INSPIRE].

[9] CMS collaboration, S. Chatrchyan et al., Search for supersymmetry at the LHC in events with jets and missing transverse energy, Phys. Rev. Lett. 107 (2011) 221804 [arXiv:1109.2352] [INSPIRE].

[10] CMS collaboration, Search for supersymmetry in all-hadronic events with missing energy, PAS-SUS-11-004.

[11] CMS collaboration, Search for supersymmetry in all-hadronic events with MT2, PAS-SUS-11-005.

[12] CMS collaboration, Search for supersymmetry in all-hadronic events with $\tau$ leptons, PAS-SUS-11-007.

[13] CMS collaboration, Search for supersymmetry with the razor variables at CMS, PAS-SUS-11-008. 
[14] CMS collaboration, Search for supersymmetry in events with photons, jets and missing energy, PAS-SUS-11-009.

[15] CMS collaboration, Search for new physics with same-sign isolated dilepton events with jets and missing energy, PAS-SUS-11-010.

[16] CMS collaboration, Search for new physics in events with opposite-sign dileptons and missing transverse energy, PAS-SUS-11-011.

[17] CMS collaboration, Multileptonic SUSY searches, PAS-SUS-11-013.

[18] CMS collaboration, Search for new physics with single-leptons at the LHC, PAS-SUS-11-015.

[19] S. Sekmen, S. Kraml, J. Lykken, F. Moortgat, S. Padhi, et al., Interpreting LHC SUSY searches in the phenomenological MSSM, JHEP 02 (2012) 075 [arXiv:1109.5119] [INSPIRE].

[20] A. Arbey, M. Battaglia and F. Mahmoudi, Implications of LHC searches on SUSY particle spectra: the pMSSM parameter space with neutralino dark matter, Eur. Phys. J. C 72 (2012) 1847 [arXiv:1110.3726] [INSPIRE].

[21] M. Papucci, J.T. Ruderman and A. Weiler, Natural SUSY endures, arXiv:1110.6926 [INSPIRE].

[22] B. Allanach, T. Khoo and K. Sakurai, Interpreting a $1 \mathrm{fb}^{-2}$ ATLAS search in the minimal anomaly mediated supersymmetry breaking model, JHEP 11 (2011) 132 [arXiv:1110.1119] [INSPIRE].

[23] U. Ellwanger, Higgs bosons in the next-to-minimal supersymmetric standard model at the LHC, Eur. Phys. J. C 71 (2011) 1782 [arXiv:1108.0157] [INSPIRE].

[24] S. AbdusSalam, B. Allanach, H. Dreiner, J. Ellis, U. Ellwanger, et al., Benchmark models, planes, lines and points for future SUSY searches at the $L H C$, Eur. Phys. J. C 71 (2011) 1835 [arXiv:1109.3859] [InSPIRE].

[25] ATLAS collaboration, G. Aad et al., Search for squarks and gluinos using final states with jets and missing transverse momentum with the ATLAS detector in sqrt(s) $=7 \mathrm{TeV}$ proton-proton collisions, Phys. Lett. B 710 (2012) 67 [arXiv:1109.6572] [https://atlas . web. cern.ch/Atlas/GROUPS/PHYSICS/PAPERS/SUSY-2011-07/] [INSPIRE].

[26] A. Djouadi, U. Ellwanger and A. Teixeira, The constrained next-to-minimal supersymmetric standard model, Phys. Rev. Lett. 101 (2008) 101802 [arXiv:0803.0253] [INSPIRE].

[27] A. Djouadi, U. Ellwanger and A. Teixeira, Phenomenology of the constrained NMSSM, JHEP 04 (2009) 031 [arXiv:0811.2699] [INSPIRE].

[28] U. Ellwanger, A. Florent and D. Zerwas, Discovering the constrained NMSSM with $\tau$ leptons at the LHC, JHEP 01 (2011) 103 [arXiv: 1011.0931] [INSPIRE].

[29] U. Ellwanger and C. Hugonie, NMSPEC: a Fortran code for the sparticle and Higgs masses in the NMSSM with GUT scale boundary conditions, Comput. Phys. Commun. 177 (2007) 399 [hep-ph/0612134] [INSPIRE].

[30] U. Ellwanger, J.F. Gunion and C. Hugonie, NMHDECAY: a Fortran code for the Higgs masses, couplings and decay widths in the NMSSM, JHEP 02 (2005) 066 [hep-ph/0406215] [INSPIRE].

[31] U. Ellwanger and C. Hugonie, NMHDECAY 2.0: An Updated program for sparticle masses, Higgs masses, couplings and decay widths in the NMSSM, Comput. Phys. Commun. 175 (2006) 290 [hep-ph/0508022] [InSPIRE]. 
[32] W. Beenakker, R. Hopker, M. Spira and P. Zerwas, Squark and gluino production at hadron colliders, Nucl. Phys. B 492 (1997) 51 [hep-ph/9610490] [InSPIRE].

[33] W. Beenakker, R. Hopker and M. Spira, PROSPINO: a program for the production of supersymmetric particles in next-to-leading order QCD, hep-ph/9611232 [INSPIRE].

[34] W. Beenakker, M. Klasen, M. Krämer, T. Plehn, M. Spira, et al., The production of charginos/neutralinos and sleptons at hadron colliders, Phys. Rev. Lett. 83 (1999) 3780 [Erratum ibid. 100 (2008) 029901] [hep-ph/9906298] [INSPIRE].

[35] J. Alwall, M. Herquet, F. Maltoni, O. Mattelaer and T. Stelzer, MadGraph 5 : going beyond, JHEP 06 (2011) 128 [arXiv: 1106.0522] [InSPIRE].

[36] T. Sjöstrand, S. Mrenna and P.Z. Skands, PYTHIA 6.4 physics and manual, JHEP 05 (2006) 026 [hep-ph/0603175] [INSPIRE].

[37] J. Alwall, S. de Visscher and F. Maltoni, QCD radiation in the production of heavy colored particles at the LHC, JHEP 02 (2009) 017 [arXiv:0810.5350] [INSPIRE].

[38] D. Das, U. Ellwanger and A.M. Teixeira, NMSDECAY: a Fortran code for supersymmetric particle decays in the next-to-minimal supersymmetric standard model, Comput. Phys. Commun. 183 (2012) 774 [arXiv:1106.5633] [INSPIRE].

[39] M. Muhlleitner, A. Djouadi and Y. Mambrini, SDECAY: a Fortran code for the decays of the supersymmetric particles in the MSSM, Comput. Phys. Commun. 168 (2005) 46 [hep-ph/0311167] [INSPIRE].

[40] S. Ovyn, X. Rouby and V. Lemaitre, DELPHES, a framework for fast simulation of a generic collider experiment, arXiv:0903.2225 [INSPIRE].

[41] M. Cacciari and G.P. Salam, Dispelling the $N^{3}$ myth for the $k_{t}$ jet-finder, Phys. Lett. B 641 (2006) 57 [hep-ph/0512210] [INSPIRE].

[42] K. Cheung and T.-J. Hou, Light pseudoscalar Higgs boson in neutralino decays in the next-to-minimal supersymmetric standard model, Phys. Lett. B 674 (2009) 54 [arXiv: 0809.1122] [INSPIRE].

[43] O. Stal and G. Weiglein, Light NMSSM Higgs bosons in SUSY cascade decays at the LHC, JHEP 01 (2012) 071 [arXiv:1108.0595] [INSPIRE].

[44] S. Kraml, A. Raklev and M. White, NMSSM in disguise: discovering singlino dark matter with soft leptons at the LHC, Phys. Lett. B 672 (2009) 361 [arXiv:0811.0011] [INSPIRE].

[45] V. Barger, G. Shaughnessy and B. Yencho, Many leptons at the LHC from the NMSSM, Phys. Rev. D 83 (2011) 055006 [arXiv:1011.3526] [INSPIRE]. 